\title{
The construction problem for Hodge numbers modulo an integer in positive characteristic-ERRATUM
}

\author{
Remy van Dobben de Bruyn ${ }^{1,2}$ and Matthias Paulsen ${ }^{3}$ \\ ${ }^{1}$ Department of Mathematics, Princeton University, Fine Hall, Washington Road, Princeton, NJ 08544, USA; \\ E-mail: rdobben@math.princeton.edu. \\ ${ }^{2}$ Institute for Advanced Study, 1 Einstein Drive, Princeton, NJ 08540, USA. \\ ${ }^{3}$ Institute of Algebraic Geometry, Gottfried Wilhelm Leibniz Universität Hannover, Welfengarten 1, D-30167 Hannover, \\ Germany; E-mail: paulsen@math.uni-hannover.de.
}

The original publication of this article includes an error introduced during the publication process. On three occasions, Remark 4.4 has been incorrectly referred to as Theorem 4.4.

Please find listed below the corrected paragraphs containing the errors in the original publication along with the page number of where they occur.

\section{Page 2:}

In analogy with [5, Theorem 2], our constructions only need Serre's counterexample [14, Proposition 16] to generate all Hodge asymmetry. While the structure of our argument is similar to [11], the absence of condition (3) in positive characteristic raises new difficulties for both the inner and outer Hodge numbers. There is a quick proof of Theorem 2 assuming embedded resolution of singularities in positive characteristic; see Remark 4.4. The proof we present is similar but does a little more work to avoid using embedded resolution. It relies on Maruyama's theory of elementary transformations of vector bundles, which we briefly recall in the Appendix.

\section{Page 9:}

Remark 4.4. The proof above can be simplified if one assumes embedded resolution of singularities in arbitrary dimension. Indeed, by blowing up a finite number of points, we may assume that $h^{1,1}(X) \equiv a^{1,1}-1(\bmod m)$ and $X$ contains $\mathbf{P}^{n-1}$. Now we claim that we can construct an $(n-2)$-dimensional subvariety $Y$ in a blowup $X^{\prime} \rightarrow X$ with $h^{p, q}\left(X^{\prime}\right) \equiv h^{p, q}(X)(\bmod m)$ such that $h^{p, q}(Y) \equiv a^{p+1, q+1}-h^{p+1, q+1}(X)(\bmod m)$. Then the blowup $\tilde{X} \rightarrow X^{\prime}$ in $Y$ has the required Hodge numbers.

To construct $Y$, first construct any smooth projective variety $Z$ of dimension $n-2$ with the correct outer Hodge numbers using Proposition 3.1. Then $Z$ is birational to a (possibly singular) hypersurface $Z^{\prime} \subseteq \mathbf{P}^{n-1}$. Embedded resolution of $Z^{\prime} \subseteq \mathbf{P}^{n-1}$ gives a birational map $X^{\prime} \rightarrow X$ such that the strict transform of $Z^{\prime}$ is smooth, so $Z^{\prime}$ has the desired outer Hodge numbers by [3, Theorem 1]. By the induction hypothesis, we may blow up further to get the inner Hodge numbers we want. Repeating this construction $m-1$ more times, as usual, gives $h^{p, q}\left(X^{\prime}\right) \equiv h^{p, q}(X)(\bmod m)$.

However, because resolution of singularities is currently unknown in positive characteristic beyond dimension 3, we have developed the above approach using embedded resolution of surfaces, Maruyama's theory of elementary transformations of projective bundles, and the fortuitous fact that the failure of Hodge symmetry is 'generated' by surfaces (see also [5, Theorem 2]). 


\section{Page 9:}

Remark 4.5. Both the proof of Theorem 2 above (replacing Lemma 4.1 with an easy case of [11, Lemma 6]) and the alternative argument of Remark 4.4 using resolution of singularities give new methods to prove the characteristic zero result [11, Theorem 5].

Conversely, it is possible to adapt the methods of $[11, \S 3]$ to prove Theorem 2, using the subvarieties from [11, Lemma 6] as well as projective bundles over the subvarieties from Lemma 4.1, but the analysis is a bit more intricate.

\section{Reference}

[1] R. Van Dobben de Bruyn and M. Paulsen, 'The construction problem for Hodge numbers modulo an integer in positive characteristic', Forum Math. Sigma 8 (2020), E45. doi:10.1017/fms.2020.48 